\title{
РЕКУРСИВНЫЙ МЕТОД ВЫЧИСЛЕНИЯ МНОГОЧАСТИЧНЫХ ФУНКЦИЙ ГРИНА
}

A. SERMAN. MITMEOSAKESELISE GREENI FUNKTSIOONI ARVUTAMISE REKURSIIVMEETOD

A. SHERMAN. A RECURSION METHOD FOR CALCULATING MANY-PARTICLE GREEN'S FUNCTIONS

\section{(Представил В. Хижняков)}

Высокая эффективность рекурсивного метода вычисления одночастичных функций Грина (ФГ, см., напр., $\left.\left[{ }^{1,2}\right]\right)$ делает весьма привлекательной задачу его использования и для многочастичных ФГ. В данном сообщении эта задача решается для ФГ вида

$$
\begin{gathered}
S\left(z_{1}, z_{2}, \Omega\right)=\int_{-\infty}^{\infty} \mathrm{dt} \int_{0}^{\infty} d \tau_{1} d \tau_{2} \exp \left(i \Omega t-z_{1} \tau_{1}-z_{2}^{*} \tau_{2}\right) S\left(\tau_{1}, \tau_{2}, t\right), \\
S\left(\tau_{1}, \tau_{2}, t\right)=\left\langle e^{i H_{L} t} a_{\mathbf{k}_{1}}\left(\tau_{1}\right) a_{\mathrm{k}_{2}}^{+} e^{-i H_{L} t} a_{\mathbf{k}_{2}} a_{\mathbf{k}_{1}}^{+}\left(\tau_{2}\right)\right\rangle,
\end{gathered}
$$

где $S\left(-i \Omega_{1}-\gamma,-i \Omega_{1}-\gamma, \Omega_{1}-\Omega_{2}\right)$ описывает спектр резонансного вторичного свечения кристалла в случае, когда частота возбуждающего света $\Omega_{1}$ попадает в резонанс с уединенной экситонной зоной, $\gamma \rightarrow+0$, $\Omega_{2}$ - частота вторичного фотона, $\mathbf{k}_{1}\left(\mathbf{k}_{2}\right)$ - волновой вектор первичного (вторичного) фотона, $a_{\mathrm{k}}^{+}$- оператор рождения экситона с волновым вектором $\mathbf{k}, a_{\mathbf{k}}(t)=\exp (i H t) a_{\mathbf{k}} \exp (-i H t), \quad H$ и $H_{L}-$ гамильтонианы экситон-фононной и фононной систем кристалла, $\langle\ldots\rangle=\mathrm{Sp}\left\{\exp \left(-H_{L} / T\right)|0\rangle\langle 0| \ldots\right\} / \mathrm{Sp}\left\{\exp \left(-H_{L} / T\right)\right\}, T-$ температура, $|0\rangle$ - вакуумное состояние экситонной системы. Сходная с приводимой ниже процедура вычисления может быть использована и для многочастичных ФГ иного вида. Случай усреднения по чистому состоянию отдельно рассматриваться не будет, поскольку, как показано в $\left[{ }^{3}\right]$, имеется полная аналогия в формулах между этим случаем и рассматриваемым случаем усреднения по каноническому ансамблю. Соответствующие результаты могут быть легко получены по аналогии с приводимыми ниже на основе формул работ $\left[{ }^{2}\right]$.

Будем далее считать, что решена задача определения операторов базиса Ланцоша-Мори $\left\{A_{n}\right\}, n=0,1,2, \ldots$, с начальным оператором $A_{0}=a_{\mathbf{k}_{1}}$ и элементов цепной дроби, представляющей одночастичную $Ф Г, E_{n}$ и $V_{n}\left[{ }^{4,3}\right]$. Из двух имеющихся вариантов решения [3] выберем тот, который ведет к ортонормированному базису: $\left\langle A_{n} A_{m}^{+}\right\rangle=$ $=\delta_{n, m}$. Определив, как и в $\left[{ }^{3}\right]$, 


$$
\begin{gathered}
\frac{d}{\mathrm{dt}} A_{n}(t)=i \prod_{m=0}^{n-1}\left(1-P_{m}\right)\left[H, A_{n}(t)\right], \quad A_{n}(0)=A_{n}, \\
P_{m} Q=\left\langle Q A_{m}^{+}\right\rangle A_{m}, \quad R_{m}(t)=\left\langle A_{m}(t) A_{m}^{+}\right\rangle
\end{gathered}
$$

формулу (6) этой работы можно представить в виде

$$
A_{0}(t)=\sum_{n=0}^{\infty} \int_{0}^{t} d s_{1} \int_{0}^{s_{1}} d s_{2} \ldots \int_{0}^{s_{n-1}} d s_{n} R_{0}\left(t-s_{1}\right) i V_{1} R_{1}\left(s_{1}-s_{2}\right) \ldots i V_{n} R_{n}\left(s_{n}\right) A_{n} .
$$

Подставив (2) в (1), находим

$$
\begin{gathered}
S\left(z_{1}, z_{2}, \Omega\right)= \\
=\sum_{n, m=0}^{\infty} R_{0}\left(z_{1}\right)\left[\prod_{j=1}^{n} i V_{j} R_{j}\left(z_{1}\right)\right] R_{0}^{*}\left(z_{2}\right)\left[\prod_{k=1}^{m}(-i) V_{k} R_{k}^{*}\left(z_{2}\right)\right] C_{n m}(\Omega), \\
C_{n m}(\Omega)=\int_{-\infty}^{\infty} \mathrm{dt} e^{i \Omega t}\left\langle e^{i H_{L} t} A_{n} a_{\mathbf{k}_{2}}^{+} e^{-i H_{L} t} a_{k_{2}} A_{m}^{+}\right\rangle .
\end{gathered}
$$

Формулы (3) и есть требуемые соотношения. Как и в $\left[{ }^{2,3}\right]$, операторы $A_{n}$ в этой задаче удобно выражать через операторы рождения и уничтожения фононов, тогда величины $C_{n m}(\Omega)$ легко вычисляются. Учитывая также, что согласно формуле (7) работы [3]

$$
R_{n}(z)=\int_{0}^{\infty} \mathrm{dt} e^{-z t} R_{n}(t)=\left[z+i E_{n}+V_{n+1}^{2} R_{n+1}(z)\right]^{-1}
$$

видим, что рекурсивный метод, предназначавшийся для вычисления одночастичных ФГ дает все необходимые средства и для вычисления многочастичных ФГ, представляя их в виде ряда (3), членами которого являются цепные дроби (4). По-видимому, наиболее существенным усложнением в рассматриваемом применении в сравнении с обычным использованием рекурсивного метода является необходимость хранения в памяти ӘВМ компонент в общем случае всех вычисляемых операторов $A_{n}$ для определения $C_{n m}(\Omega)$.

Разложение (3) по виду напоминает обычно используемые в таких задачах ряды теории возмущений по оператору экситон-фононного взаимодействия (см., напр., $\left.\left[{ }^{5}\right]\right)$, с той, однако, существенной разницей, что вместо ФГ свободных частиц в (3) входят полные $Ф Г R_{n}(z)$. В этом и в относительной простоте формул (3) и состоит основное преимущество использования рекурсивного метода для вычисления многочастичных ФГ. Как известно, для улучшения сходимости рядов теории возмущений используется процедура их частичного суммирования. Поэтому можно ожидать, что ряды типа (3) сходятся лучше рядов теории возмущений. Применительно к рассматриваемой задаче быстрая сходимость ряда (3) следует из результатов [ $\left.{ }^{2}\right]$.

В качестве примера использования полученных формул рассмотрим модель ориентированного газа, описываемую гамильтонианом

$$
H=\sum_{\mathrm{m}}\left\{\varepsilon a_{\mathrm{m}}^{+} a_{\mathrm{m}}+\omega b_{\mathrm{m}}^{+} b_{\mathrm{m}}+\sqrt{S \omega} a_{\mathrm{m}}^{+} a_{\mathrm{m}}\left(b_{\mathrm{m}}+b_{\mathrm{m}}^{+}\right),\right.
$$

где $a_{\mathrm{m}}^{+}=N^{-1 / 2} \sum_{\mathbf{k}} \exp (i \mathrm{~km}) a_{\mathrm{k}}^{+}$и $b_{\mathrm{m}}^{+}$- операторы рождения возбуждения и фонона на узле $m$ решетки, $N$ - число узлов в периодической области кристалла, $\varepsilon, \omega$ и $S-$ энергия терма, частота колебаний и стоксовы потери соответственно. При $T=0\left[{ }^{2}\right]$ 


$$
\check{E}_{n}=\varepsilon+n \omega, \quad V_{n}^{2}=S \omega n, \quad A_{n}=(N n !)^{-1 / 2} \sum_{\mathrm{m}} e^{i \mathbf{k}_{1} \mathrm{~m}} b_{\mathrm{m}}^{n} a_{\mathrm{m}},
$$

и из (3) находим

$$
\begin{gathered}
S\left(z, z, \Omega_{1}-\Omega_{2}\right)=\left|R_{0}(z)\right|^{2} \delta_{\mathbf{k}_{1}, \mathbf{k}_{2}} \delta\left(\Omega_{1}-\Omega_{2}\right)+ \\
+N^{-1}\left|R_{0}(z)\right|^{2} \sum_{n=1}^{\infty}\left[\prod_{k=1}^{n} V_{k}^{2}\left|R_{k}(z)\right|^{\mathbf{a}}\right] \delta\left(\Omega_{1}-\Omega_{2}-n \omega\right)
\end{gathered}
$$

где $z=-i \Omega_{1}-\gamma$, а $R_{n}(z)$ определяется формулами (4), (5).

В случае использования матричного рекурсивного метода $\left[{ }^{6}\right]$ формула (2) сохраняет свой вид, если под $R_{n}(t), V_{n}$ и $A_{n}$ понимать соответствующие матрицы. Аналогичным изменениям подвергается и формула (3).

\section{Л ИТЕ РАТ У РА}

1. Haydock, R. Solid State Phys., 35, 216-294 (1980); Kelly, M. J. Solid State Phys., 35, 295-383 (1980); The Recursion Method and Its Applications (eds D. G. Pettifor, D. L. Weaire). Berlin-Heidelberg-New York-Tokyo, Springer Verlag, 1985.

2. Sherman, A. V. Phys. status solidi (b), 135, № 2, 697-705 (1986); 141, № 1, 151161 (1987).

3. Sherman, A. V. J. Phys. A, 20, № 3, 569-576 (1987).

4. Mori, H. Progr. Theor. Phys., 34, № 3, 399-416 (1965).

5. Hizhnyakov, V. V., Sherman, A. V. Phys. status solidi (b), 85, № 1, 51-61 (1978).

6. Шерман А. Изв. АН ЭстССР. Физ. Матем., 37, (1988).

$\begin{array}{cc}\text { Институт физики } & \text { Поступила в редакцию } \\ \text { Академии наук Эстонской ССР } & 28 / \mathrm{IX} \\ & 1987\end{array}$

